\title{
A Foreshock Model for Interstellar Shocks of Solar Origin: Voyager 1 and 2 Observations
}

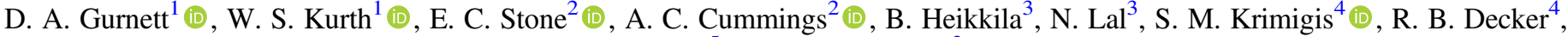 \\ N. F. Ness ${ }^{5}$, and L. F. Burlaga ${ }^{3}$ iD \\ ${ }^{1}$ University of Iowa, Department of Physics and Astronomy, Iowa City, IA 52242, USA; donald-gurnett@uiowa.edu \\ ${ }^{2}$ California Institute of Technology, 1200 East California Boulevard, Pasadena, CA 91125, USA \\ ${ }_{4}^{3}$ NASA Goddard Space Flight Center, 8800 Greenbelt Road, Greenbelt, MD 20771, USA \\ ${ }^{4}$ Applied Physics Laboratory/JHU, 11100 Johns Hopkins Road, Laurel, MD 20723, USA \\ ${ }^{5}$ University of Delaware, Newark, DE 19716, USA \\ Received 2020 August 31; revised 2020 October 19; accepted 2020 October 19; published 2020 December 3
}

\begin{abstract}
The Voyager 1 (V1) and Voyager 2 (V2) spacecraft were launched in 1977 on a mission to explore the outer planets and reach the heliopause, the boundary between the hot solar plasma and the relatively cool interstellar plasma. V1 reached the heliopause on 2012 August 25, at 121.6 au, and V2 reached the heliopause on 2018 November 5, at $119.0 \mathrm{au}$. One of their remarkable discoveries was the detection of shocks propagating into the interstellar plasma from energetic solar events. These shocks are typically preceded by electron plasma oscillations excited by electron beams streaming along interstellar magnetic field lines ahead of the shocks. The frequencies of the plasma oscillations have now provided radial electron density profiles in the outer heliosphere and in the interstellar medium to radial distances of more than $145 \mathrm{au}$. The oscillations are typically preceded by bursts of high-energy $\sim 5-100 \mathrm{MeV}$ electrons. These electron bursts are interpreted as being due to the reflection (and acceleration) of cosmic-ray electrons by the shock at the time the shock first contacts the magnetic field line that passes through the spacecraft. Relative timing between the cosmic rays reflected by the shock and the onset of the plasma oscillations allow us, for the first time, to estimate the energy, $\sim 20-100 \mathrm{eV}$, of the electron beams responsible for the plasma oscillations. These observations are combined into a self-consistent model called the foreshock model that describes the interaction of shocks of solar origin with the interstellar plasma.
\end{abstract}

Unified Astronomy Thesaurus concepts: Interstellar medium (847)

\section{Introduction}

After completing their exploration of the outer planets, the Voyager 1 (V1) and Voyager 2 (V2) spacecraft, both launched in 1977, continued traveling outward from the Sun on a mission to reach the heliopause, which separates the hot, $10^{5}-10^{6} \mathrm{~K}$, heliospheric plasma from the much cooler, $\sim 10^{4} \mathrm{~K}$, interstellar plasma (Davis 1955; Parker 1963, pg. 138; Axford 1990; Zank 2015). V1 crossed the heliopause on 2012 August 25 at a heliocentric radial distance of 121.6 au (Burlaga et al. 2013b; Gurnett et al. 2013; Krimigis et al. 2013; Stone et al. 2013), and V2 crossed the heliopause on 2018 November 5 at a radial distance of 119.0 au (Burlaga et al. 2019; Gurnett \& Kurth 2019; Krimigis et al. 2019; Richardson et al. 2019; Stone et al. 2019). The trajectories of the two spacecraft, and the locations of the heliopause crossings, are given in Figure 1. Having reached the interstellar medium, often now referred to as the very local interstellar medium (VLISM), it was soon discovered that shocks propagating outward from energetic disturbances at the Sun were continuing into the VLISM (Burlaga et al. 2013a; Gurnett et al. 2015). The possibility of shocks of solar origin propagating into the interstellar plasma was first suggested by Gurnett et al. (1993) to explain the generation of transient $2-3 \mathrm{kHz}$ heliospheric radio emissions, which often had increasing frequencies lasting nearly a year after the onset of the event. The $2-3 \mathrm{kHz}$ radio emissions were first detected (Kurth et al. 1984) by the V1 and V2 plasma wave (PWS) instruments while still well inside the heliosphere, shortly after the flybys of Saturn over 35 years ago. According to the current widely accepted explanation, these radio emissions are produced by electron plasma oscillations driven by electron beams upstream of shocks as they propagate through the interstellar plasma (Gurnett et al. 1993; Cairns \& Zank 2000). The radio emissions are believed to be generated from the plasma oscillations via nonlinear mode conversion processes, like those known to be responsible for Type II solar radio bursts (Wild et al. 1954; Ginzburg \& Zheleznyakov 1958; Reiner et al. 1998; Bale et al. 1999). The increase in the radio emission frequency with increasing time after the onset of the event is attributed to the increase in the electron density, hence plasma oscillation frequency $\left(f_{\mathrm{p}}=8980 \sqrt{ } n_{\mathrm{e}} \mathrm{Hz}\right.$, where $n_{\mathrm{e}}$ in $\mathrm{cm}^{-3}$ is the electron density), as the shock propagates into the interstellar plasma. Since the electron beam and plasma oscillations occur upstream of the shock, these beams and the associated plasma oscillations constitute precursors to interstellar shocks propagating outward from the Sun. A more detailed description of the $2-3 \mathrm{kHz}$ radio emission mechanism is given in Gurnett et al. (2015), together with an initial discussion of various other precursor effects detected by V1. This current paper is essentially a sequel to the Gurnett et al. (2015) paper using the much more extensive data now available from both V1 and V2.

\section{Plasma Oscillations and the Local Plasma Density}

Electron plasma oscillations, and radio emissions, are detected by the V1 PWS using two $10 \mathrm{~m}$ electric antenna elements that are mounted in a V-shaped configuration on the spacecraft (Scarf \& Gurnett 1977). Although designed for polarization measurements by the planetary radio astronomy (PRA) instrument (Warwick et al. 1977), the PWS uses the two antennas as an electric dipole 


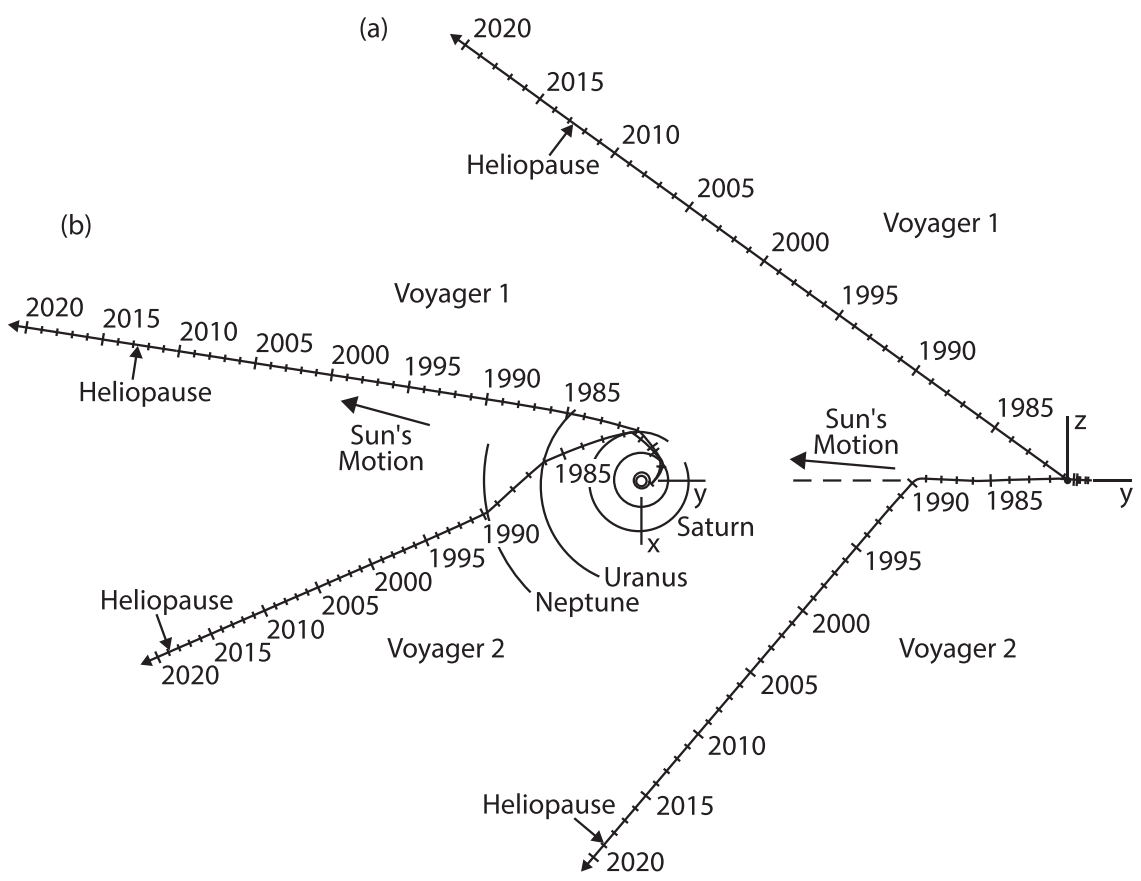

Figure 1. The trajectories of V1 and V2 in inertial $(x, y, z)$ coordinates as a function of year since their launch in 1977. Panel (a) is the projection onto the $x-z$ plane, and panel (b) is the projection onto the $x-y$ plane. The locations of the crossings of the heliopause, the boundary between the solar plasma and the interstellar plasma, are marked.

antenna with an effective length of $7 \mathrm{~m}$. Two methods are used to process signals from the antenna. In the first method, antenna voltage waveforms are sampled by a wideband receiver at a rate of 28,800 samples per second with 4 bit resolution over a frequency range from $50 \mathrm{~Hz}$ to $10 \mathrm{kHz}$. These high rate samples are stored in the onboard digital tape recorder for later transmission to the ground. During the interstellar phase of the mission, this waveform sampling usually consists of one $48 \mathrm{~s}$ waveform every week, with subsequent playbacks of the recorded data approximately once every three months. On the ground the waveforms are subsequently Fourier transformed into 800-point frequency spectrums and plotted versus time as a color coded frequency-time spectrogram, as shown in the top panel of Figure 2. The color indicates the intensity of the signal, with red being the most intense and blue being the least intense. In the second method, the electric field waveforms are processed by an onboard 16-channel spectrum analyzer that has a frequency resolution of four channels per decade from $10 \mathrm{~Hz}$ to $56 \mathrm{kHz}$. The filter bandwidths are $\pm 15 \%$ for the lowest 8 channels, and $\pm 7.5 \%$ for the highest 8 channels. With this system the high rate tape-recorded waveforms have by far the best frequency resolution, typically about $1 \%$, but have the disadvantage of providing only one spectrum per week, whereas the 16-channel onboard spectrum analyzer data have much better time resolution, one 16-channel spectrum every $16 \mathrm{~s}$, but much poorer frequency resolution. Unfortunately, the PWS wideband receiver on V2 failed and was turned off in 2006, so only the 16-channel spectrum analyzer data are currently available from V2.

During the time since V1 crossed the heliopause, the V1 PWS has detected eight distinct electron plasma oscillation events, each of which is believed to be associated with a shock propagating outward from the Sun. These eight events can be seen in panel (a) of Figure 2. Of these, only two events have been directly associated with shocks, as identified by the spacecraft magnetometer (MAG). The two black vertical dashed lines labeled "shock" in panel (b) show jumps in the magnetic field strength that are characteristic of shocks. See Burlaga et al. (2013a) and Gurnett et al. (2015) for detailed analyses of these shocks. Plasma oscillation events for which no magnetic field signature of a shock was detected are believed to be produced by magnetic-field-aligned electron beams from distant shocks that did not pass close enough to the spacecraft to produce characteristic jumps in the magnetic field strength.

The oscillation frequency for the 2012 October-November plasma oscillation event provided the first definitive confirmation that V1 crossed the heliopause into the interstellar medium on or about 2012 August 25 (see the arrow marked "heliopause" at the top left of Figure 2). The electron plasma density inferred for this first event, $n_{\mathrm{e}} \sim 0.055 \mathrm{~cm}^{-3}$ (based on $f_{\mathrm{p}}=8980 \sqrt{ } n_{\mathrm{e}} \mathrm{Hz}$ ), can be read from the scale on the right-hand side of the spectrogram. This density was consistent with the plasma density expected in the VLISM from contemporary ground-based remote sensing measurements at that time (Frisch et al. 2011). The steady increase in the plasma frequency and electron density from the 2012 October-November event to the 2013 April-May event has been shown to be consistent with the plasma density ramp inferred from the characteristic increasing frequency of the $2-3 \mathrm{kHz}$ radio emissions after the onset of the event (Gurnett et al. 2013). For the possible origin of this steep density gradient, now called the heliospheric boundary layer, see the discussions by Baranov \& Malama (1993), Fuselier \& Cairns (2013), and Pogorelov et al. (2017).

Except possibly for the most recent 2019 May-June event, the frequencies of the plasma oscillations in panel (a) of Figure 2 show that the interstellar plasma density has slowly increased as the spacecraft moved farther into the VLISM, beyond the initial density ramp near the heliopause. This general trend for the plasma density to increase with increasing radial distance from the Sun is shown in more detail by the black dots in Figure 3, which give the average electron densities derived from the plasma oscillation events in panel (a) 


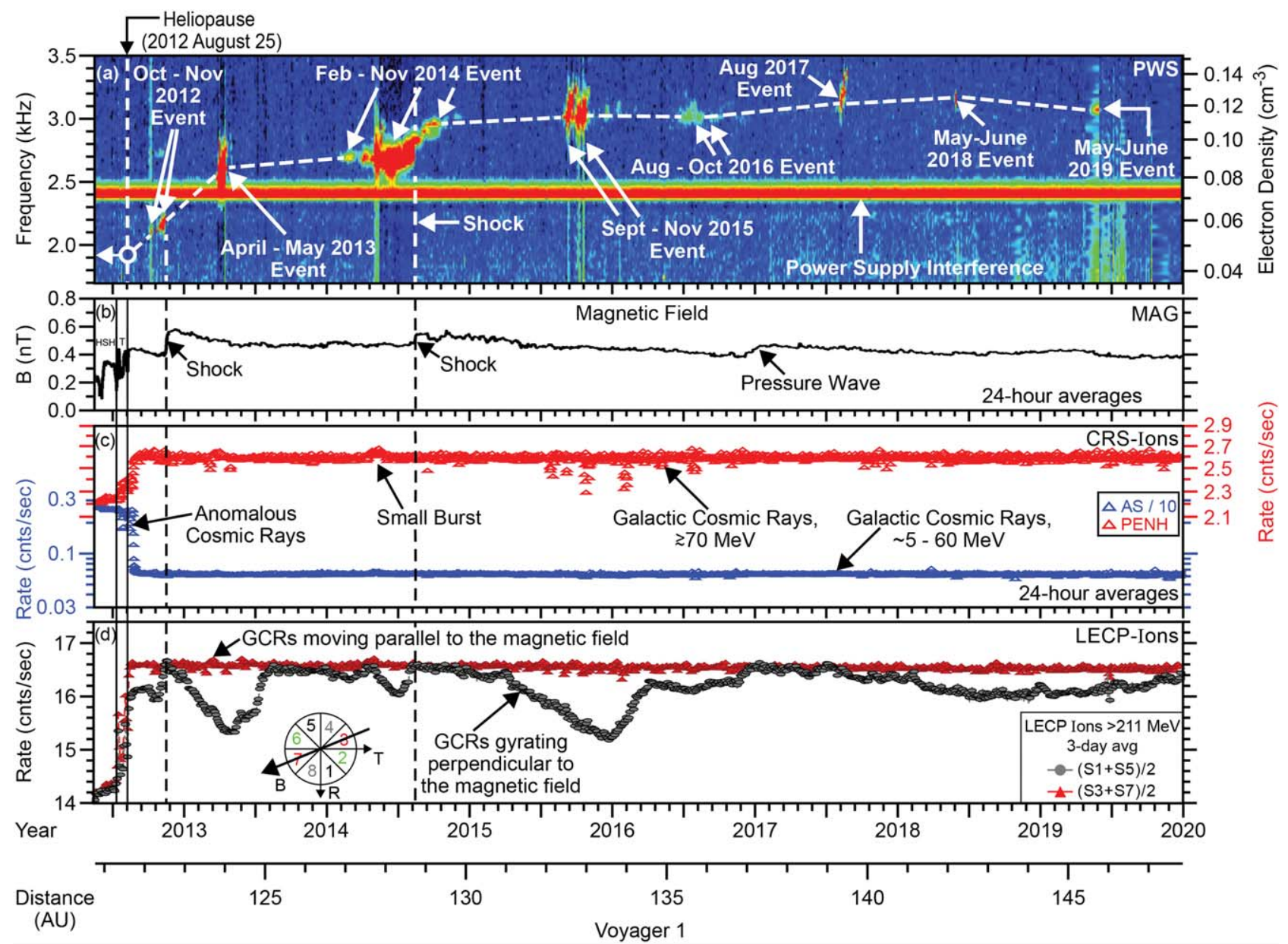

Figure 2. An overview of particle and field measurements from V1. The location of the heliopause crossing is shown by the arrow in the upper left-hand corner. (a) A color coded frequency-time spectrogram of electric field intensities from the plasma wave (PWS) wideband receiver. Red indicates high intensities, and blue indicates low intensities. (b) Magnetic field strengths from the magnetometer (MAG) in nanotesla (nT). (c) The counting rates (mainly protons) from the cosmic-ray (CRS) instrument in two energy ranges, $\gtrsim 70 \mathrm{MeV}$ (red) and $\sim 5-60 \mathrm{MeV}$ (blue). The abrupt decrease in the anomalous cosmic-ray intensities near the heliopause (HP) is evident, as is the corresponding increase in the energetic, $\gtrsim 70 \mathrm{MeV}$, galactic cosmic rays. (d) The counting rates from the low-energy charged particle (LECP) instrument for galactic cosmic rays (GCRs), mainly protons with energies $>211 \mathrm{MeV}$, propagating parallel (red) and perpendicular (gray) to the magnetic field.

of Figure 2. This illustration also shows electron densities derived from V1 plasma oscillations observed near the termination shock (TS) crossing around 94 au (black dots with error bars). No wideband high rate waveforms were available around the TS crossing, so the only frequency measurements available were from the 16-channel spectrum analyzer, which accounts for the large error bars. Note the large density increase from the TS to the interstellar plasma, about a factor of 50 (indicated by the black dashed line). This large density jump is a consequence of pressure balance between the hot plasma inside the heliosphere and the relatively much cooler plasma in the interstellar medium. Also shown are the electron densities (red dots with error bars) obtained from two recent plasma oscillation events detected in the VLISM by the V2 PWS (Gurnett \& Kurth 2019; Kurth \& Gurnett 2020), the first in the $1.78 \mathrm{kHz}$ channel at $119.7 \mathrm{au}$, and the second in the $3.11 \mathrm{kHz}$ channel at $124.2 \mathrm{au}$. The error bars for these points are larger than for the V1 interstellar densities (black dots) because, due to the failure of the wideband receiver on V2, these measurements came from the 16-channel spectrum analyzer. Despite the more limited resolution compared to that of the V1 PWS instrument the V2 measurements clearly show the steep density increase in the VLISM immediately beyond the heliopause (i.e., the density ramp), a gradient that is comparable to that observed by V1 almost eight years earlier.

\section{Cosmic-ray Precursors}

From the above, it is clear that shocks propagating into the interstellar medium are almost always preceded by strong electron plasma oscillation precursors, and that the plasma oscillations are most likely driven by low-energy electron beams streaming outward along magnetic field lines ahead of the shocks. Despite the compelling arguments supporting the electron beam generation mechanism, the charged particle instruments on V1 have not been able to confirm the existence of these electron beams, despite significant attempts to find them. This failure is partly because the V1 plasma instrument (Bridge et al. 1977), which might have been able to detect the beams, failed in 1980, and also because the energy range, $>28 \mathrm{keV}$, of the V1 low-energy charged particle (LECP) instrument (Krimigis et al. 1977) does not extend low enough to detect such beams. Studies of electron plasma oscillations upstream of Earth's bow shock (Filbert \& Kellogg 1979), and also ahead of shocks responsible for Type II solar radio emissions (Bale et al. 1999), show that the beam energies are typically from a few hundred electron volts to several 


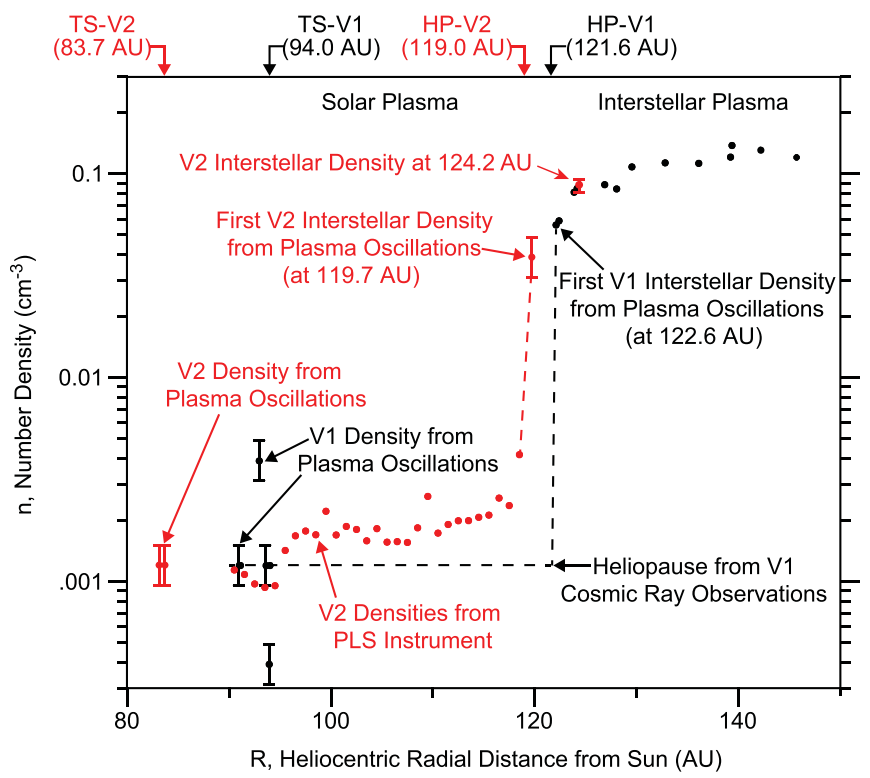

Figure 3. The plasma densities vs. heliocentric radial distance from the V1 and V2 PWS. The locations of the termination shock (TS) and heliopause (HP) crossings for the two spacecraft are indicated by the arrows at the top. The black dots are the average electron densities inferred from the oscillation frequencies of plasma oscillations detected by V1 in panel (a) of Figure 1. The red dots with error bars are the corresponding electron densities obtained from plasma oscillations detected by the V2 PWS using the 16-channel spectrum analyzer. The red dots (with no error bars) are proton densities from the V2 plasma instrument (PLS). In the proton-dominated interstellar plasma the electron and proton densities are expected to be essentially the same.

kiloelectron volts. To try to gain further understanding of the mechanism involved in the generation of the upstream plasma oscillations, we have undertaken a detailed search for other shock precursor effects in the data from the V1 cosmic-ray (CRS) instrument (Stone et al. 1977) and the LECP instrument.

Panel (c) of Figure 2 shows the CRS cosmic-ray intensities (mostly protons) in two energy ranges, $\gtrsim 70 \mathrm{MeV}$ (red) and $\sim 5-60 \mathrm{MeV}$ (blue). The abrupt increase in the galactic cosmicray (GCR) intensities (red) and the decrease in the anomalous cosmic-ray intensities (blue) are evident near the heliopause crossing. Panel (d) of Figure 2 shows the LECP intensities, consisting mostly of $>211 \mathrm{MeV}$ GCR protons propagating parallel (red) and perpendicular (gray) to the magnetic field. Note that the count rate scales have been greatly expanded to help identify possible precursor effects. Except for small statistical fluctuations, the counting rate of the GCRs in the CRS data appear to be almost completely constant. However, careful inspection shows that there are occasionally small enhancements in the intensities of the energetic $\gtrsim 70 \mathrm{MeV}$ GCRs. One such increase is marked as "small burst" in panel (c) of Figure 2. In the LECP data of panel (d) there are also sometimes similar small bursts, although they are difficult to identify. More apparent in the LECP data are the irregular occurrences of sawtooth-shaped depressions in the counting rate of GCRs gyrating perpendicular to the local magnetic field (the gray points). Such sawtooth-shaped depressions are not present in the counting rate of GCRs moving parallel to the magnetic field (red points), so they are clearly only associated with particles moving nearly perpendicular to the magnetic field. Since the trajectories of particles with pitch angles near $90^{\circ}$ are highly sensitive to small variations in the magnetic field strength, our interpretation is that these particles are somehow responding to small magnetic field variations caused by the interstellar shocks, although we do not have a detailed understanding of the systematics of these variations. In some cases, the sawtooth-shaped depressions appear to be correlated with upstream plasma oscillation events, such as near the two vertical dashed lines marked "shock." However, in other cases, especially in the more recent data, the relationship between the electron plasma oscillations and the sawtooth-shaped depressions is not obvious. For a further discussion of the origin of these depressions in the GCRs propagating perpendicular to the magnetic field and their relationship to interstellar shocks see Rankin et al. (2019) and Zhang \& Pogorelov (2020).

Upon further examination of the CRS cosmic-ray data, we discovered that the electron plasma oscillation events are consistently preceded by small bursts in the counting rates of electrons from the Electron Telescope (TET) of the CRS, a telescope which was specifically designed to detect $\gtrsim 3 \mathrm{MeV}$ electrons (Stilwell et al. 1979). An example of one such burst is illustrated in Figure 4, which shows a series of expanded timescale plots for the 2014 February-November electron plasma oscillation event. Panel (a) shows the intensity of electron plasma oscillations in the $3.11 \mathrm{kHz}$ channel of the 16 channel spectrum analyzer, panel (b) shows the magnetic field jump caused by the shock responsible for this event, panel (c) shows the $\sim 5-100 \mathrm{MeV}$ electron counting rate from TET, and panel (d) shows the G1 guard rate of the CRS HET1 highenergy telescope. The counting rate of the $\sim 5-100 \mathrm{MeV}$ electrons clearly shows that there is a burst of high-energy relativistic electrons (hatched) preceding the electron plasma oscillations by approximately 22 days. Although the HET1 G1 guard rate in panel (d) responds to a variety of particle types and energies, this Guard Rate clearly shows a nearly identical response (hatched) to the relativistic $\sim 5-100 \mathrm{MeV}$ electron burst detected by the TET. We interpret this burst of relativistic electrons as being due to the remote reflection of cosmic-ray electrons by the shock at the time that the shock first contacts the magnetic field line that passes through the spacecraft. The possibility of such a shock reflection (and acceleration) process was first suggested and analyzed by Jokipii \& Kota (2014) to explain cosmic-ray ion enhancements observed by V1 during a somewhat similar event in 2013. Their model assumes approximate conservation of the first adiabatic invariant as the particles are reflected by the increase in the magnetic field strength at the shock. Similar reflection (and acceleration) processes are also expected for energetic electrons, although the gyro radii and scale lengths involved in the interaction with the shock are quite different.

From panel (e) of Figure 4 one can also see that there are small enhancements (hatched) in the intensities in the LECP channels that are normally interpreted as being due to $>211 \mathrm{MeV}$ protons. As can be seen from the red, green, and gray points, these intensity enhancements occur almost simultaneously over a broad range of pitch angles, from nearly parallel to nearly perpendicular to the local magnetic field. Such nearly simultaneous enhancements cannot occur over such a wide range of pitch angles unless there is a large change in the local magnetic field strength, of which none is observed. Our interpretation of this enhancement, based on the simultaneous detection of $\sim 5-100 \mathrm{MeV}$ electrons by the CRS TET and the nearly simultaneous increases in the LECP count rates over a broad range of pitch angles, is that these LECP enhancements are not due to GCR protons, but rather due to the 


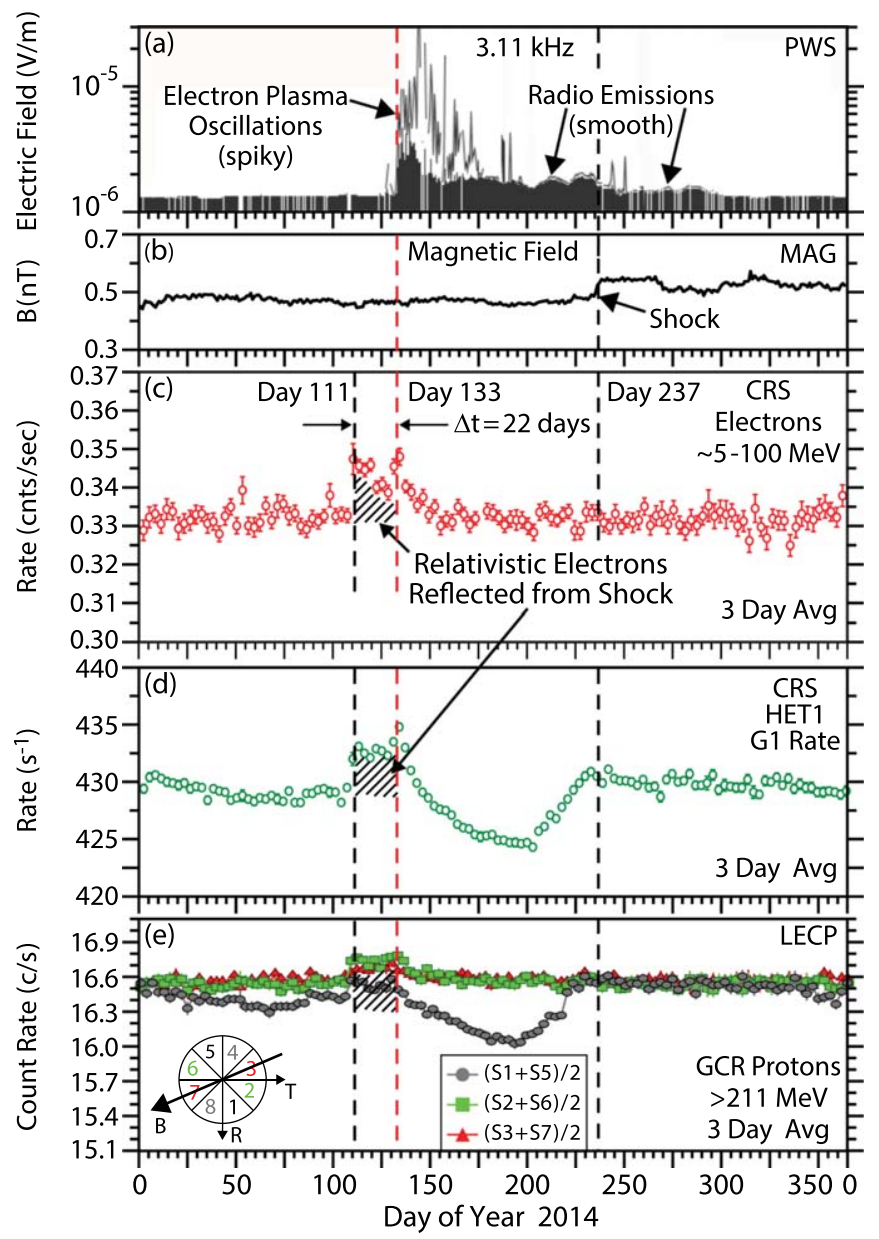

Figure 4. Upstream precursor effects associated with the 2014 FebruaryNovember electron plasma oscillation event. (a) The electric field intensities from the $3.11 \mathrm{kHz}$ channel of the PWS onboard 16-channel spectrum analyzer. Note the very abrupt onset of electron plasma oscillations on day 133, well ahead of the magnetic field jump (b) associated with the interstellar shock on day 237, as detected by the V1 magnetometer (MAG). (c) The counting rate of electrons with energies of $\sim 5-100 \mathrm{MeV}$ from the CRS TET. The enhanced intensities (hatched) are believed to be due to cosmic-ray electrons reflected (and accelerated) by the shock when the shock first contacts the magnetic field line through the spacecraft. (d) The counting rate from the CRS guard rate (G1) channel of the HET1 high-energy telescope. Although this channel responds to a variety of particle types and energies, it clearly responds (hatched region) to the relativistic electron bursts detected in panel (c). (e) The counting rates for $>211 \mathrm{MeV}$ galactic cosmic rays (mainly protons) at three different pitch angles (red, green, and gray) as detected by the LECP instrument. The enhanced count rates indicated by the hatching in the LECP data are believed to be due to the 5-100 MeV cosmic-ray electrons detected in panel (c).

burst of relativistic energetic cosmic-ray electrons penetrating the LECP solid-state detectors.

Having identified the relativistic $\sim 5-100 \mathrm{MeV}$ electron precursor to the 2014 February-November event, we next investigated all the electron plasma oscillation events detected by V1 to search for similar relativistic electron precursor events. This investigation revealed that almost all the electron plasma oscillation events detected by V1 were preceded by relativistic electron bursts comparable to that illustrated in panel (c) of Figure 4. This relationship is illustrated in Figure 5 which shows a series of relativistic electron bursts (top panel) in the counting rate from the G1 guard rate channel of HET1, and the electric field intensity (bottom panel) of the electron plasma oscillations detected in the $3.11 \mathrm{kHz}$ channel of the $\mathrm{V} 1$ PWS. The count rate in the G1 guard rate channel of HET1 was used for identifying the relativistic electron bursts because we found this channel gave better time resolution for resolving the relativistic electron bursts (vertical dashed lines) than the TET, which has a much lower average counting rate. As can be seen, five out of the eight electron plasma oscillation events were preceded by well-defined relativistic electron bursts, bursts that we interpret as being due to cosmic-ray electrons remotely reflected (and accelerated) by approaching shocks. The delay time from the first contact with the shock to the onset of the plasma oscillations was found to range from 17 to 30 days. This relationship has recently been further supported by the discovery in the recent V2 data of a very well-defined burst of 5-100 MeV electrons preceding an electron plasma oscillation event detected by the PWS on day 179, 2020 (Kurth \& Gurnett 2020). This relationship is illustrated in Figure 6 which shows that the relativistic electron burst preceded the plasma oscillations by 13 days, slightly less than the minimum delay time found for the similar V1 events in Figure 5 (17-30 days).

\section{The Interstellar Foreshock Model}

Before proceeding with a further analysis of precursor effects observed ahead of interstellar shocks, it is useful to condense what has already been learned into a tentative model that we call the interstellar foreshock model. The basic features of this model are illustrated in Figure 7 that shows a shock, the solid curved black line, propagating outward through the nearby interstellar plasma. This model was first introduced by Gurnett et al. (2015) and was adapted from the model developed by Filbert \& Kellogg (1979) to explain the generation of electron plasma oscillations by electron beams upstream of Earth's bow shock. Although similar in concept, there are three essential differences between the terrestrial foreshock model and the interstellar foreshock model. First, in the case of Earth's bow shock, the geometry is time stationary, whereas for an interstellar shock the geometry is dynamic, with the shock moving increasingly farther into the interstellar medium as time advances. Second, the scale size is much different, with the interstellar shock being some 10,000 times larger than Earth's bow shock. Third, the terrestrial bow shock model does not involve significant interactions with energetic cosmic rays. In both cases electron heating at the shock produces low-energy electrons that stream outward in front of the shock, thereby forming a beam as indicated by the arrow marked "electron beam" in Figure 7. This beam is not a monoenergetic beam, but rather is a beam in the plasma physics sense, consisting of a region of positive slope in the reduced electron velocity distribution function. The reduced distribution function is a one-dimensional velocity distribution function formed by integrating over velocities perpendicular to the magnetic field. A region of positive slope is always formed in the reduced distribution function because at any given point in the upstream region there is always a cutoff velocity below which electrons escaping from the shock cannot reach that point in space because of time-of-flight considerations (Filbert \& Kellogg 1979). It is this region of positive slope that drives electron plasma oscillations, i.e., via the beam-plasma instability (Gurnett \& Bhattacharjee 2017). The region in which electrons escaping upstream along magnetic field lines from the shock lead to the excitation of electron plasma oscillations is called the electron foreshock, see Figure 7. By analogy, because of the discovery of cosmic-ray electron and ions reflected (and accelerated) by the shock, we have introduced the idea of a 

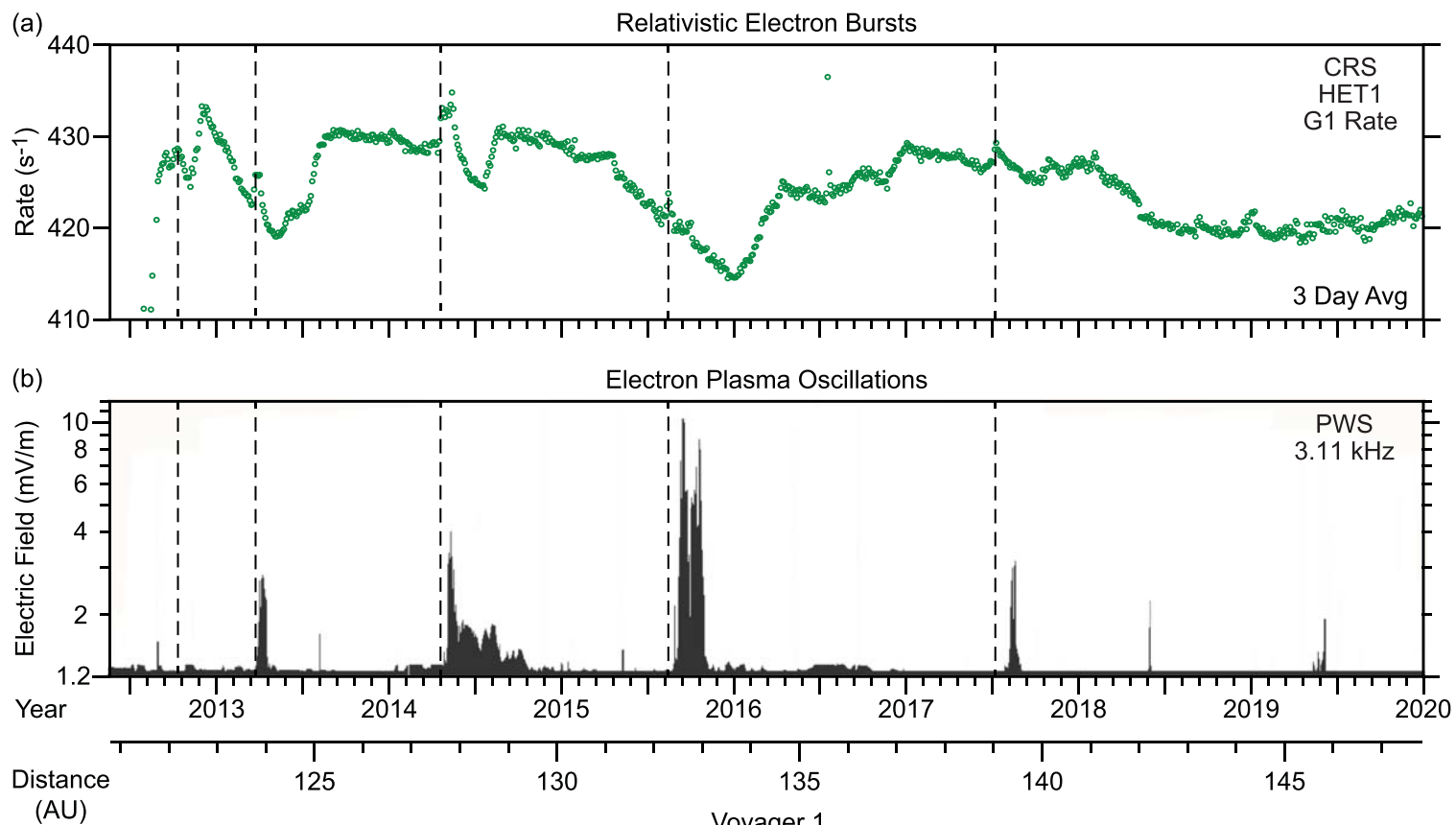

Figure 5. A comparison of the occurrence of relativistic electron bursts detected by the CRS and electron plasma oscillations detected by the PWS on V1. The top panel shows the counting rate from the G1 guard rate channel of the HET1 high-energy telescope. The bottom panel shows the electric field intensities of electron plasma oscillations in the $3.11 \mathrm{kHz}$ channel of the PWS 16-channel spectrum analyzer. The onset of the relativistic electron bursts (vertical dashed lines) consistently precede the onset of the electron plasma oscillations by about 17-30 days.

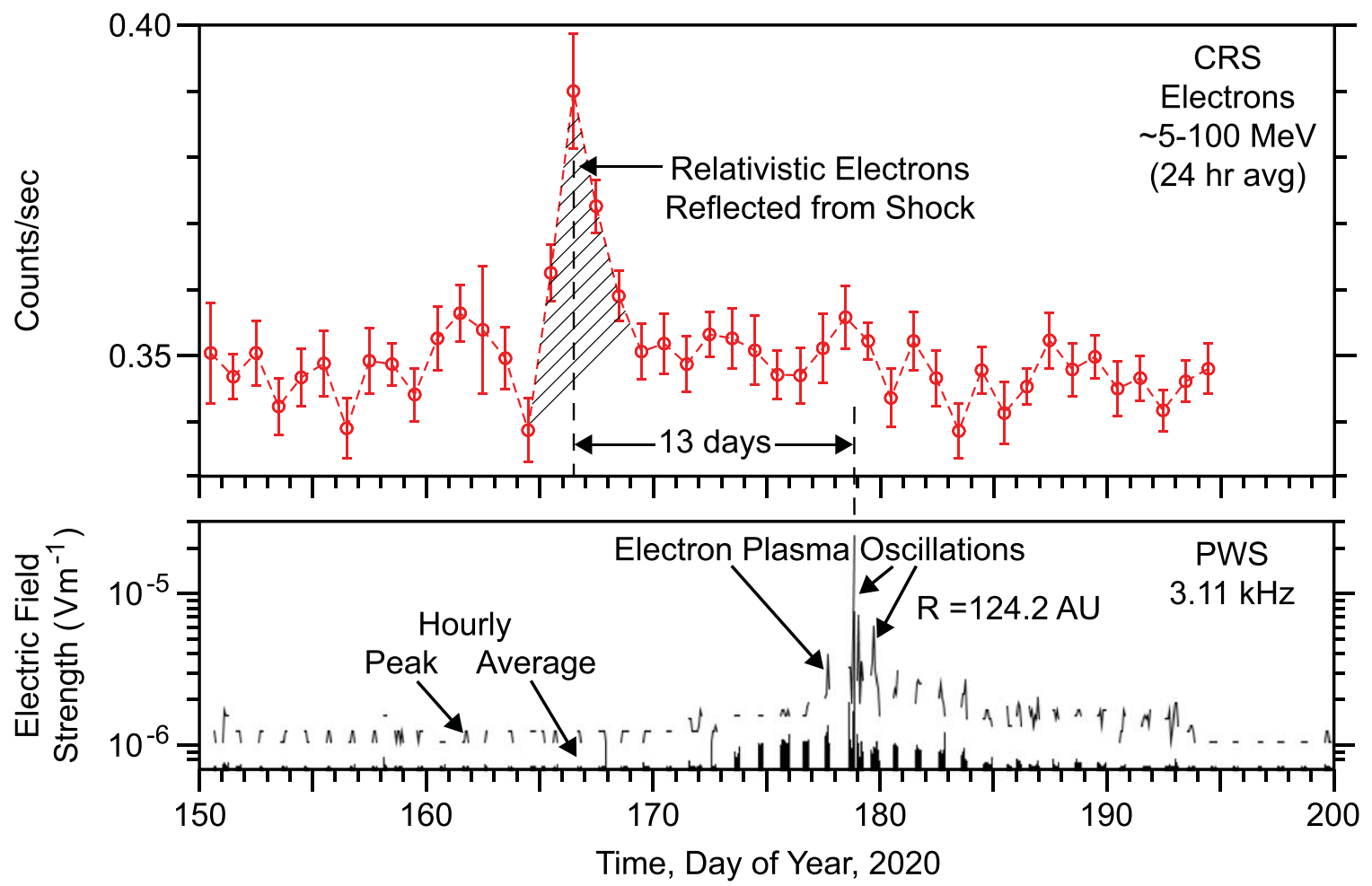

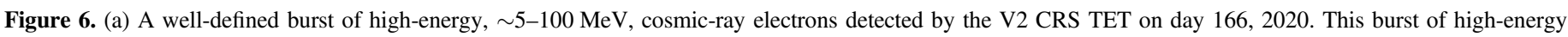

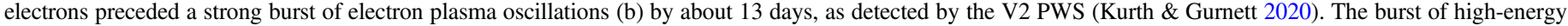

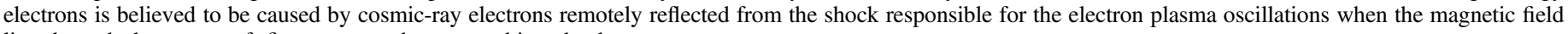
line through the spacecraft first contacts the approaching shock.

cosmic-ray foreshock. The cosmic-ray foreshock lies just behind the magnetic field line tangent to the shock, and well ahead of the electron foreshock due to the much higher velocities of reflected cosmic rays, as illustrated in Figure 7.
Although the upstream extent of the electron foreshock and the cosmic-ray foreshock are shown as distinct boundaries in Figure 7, the locations of these boundaries with respect to the tangent magnetic line are not fixed, but depend on the velocities 


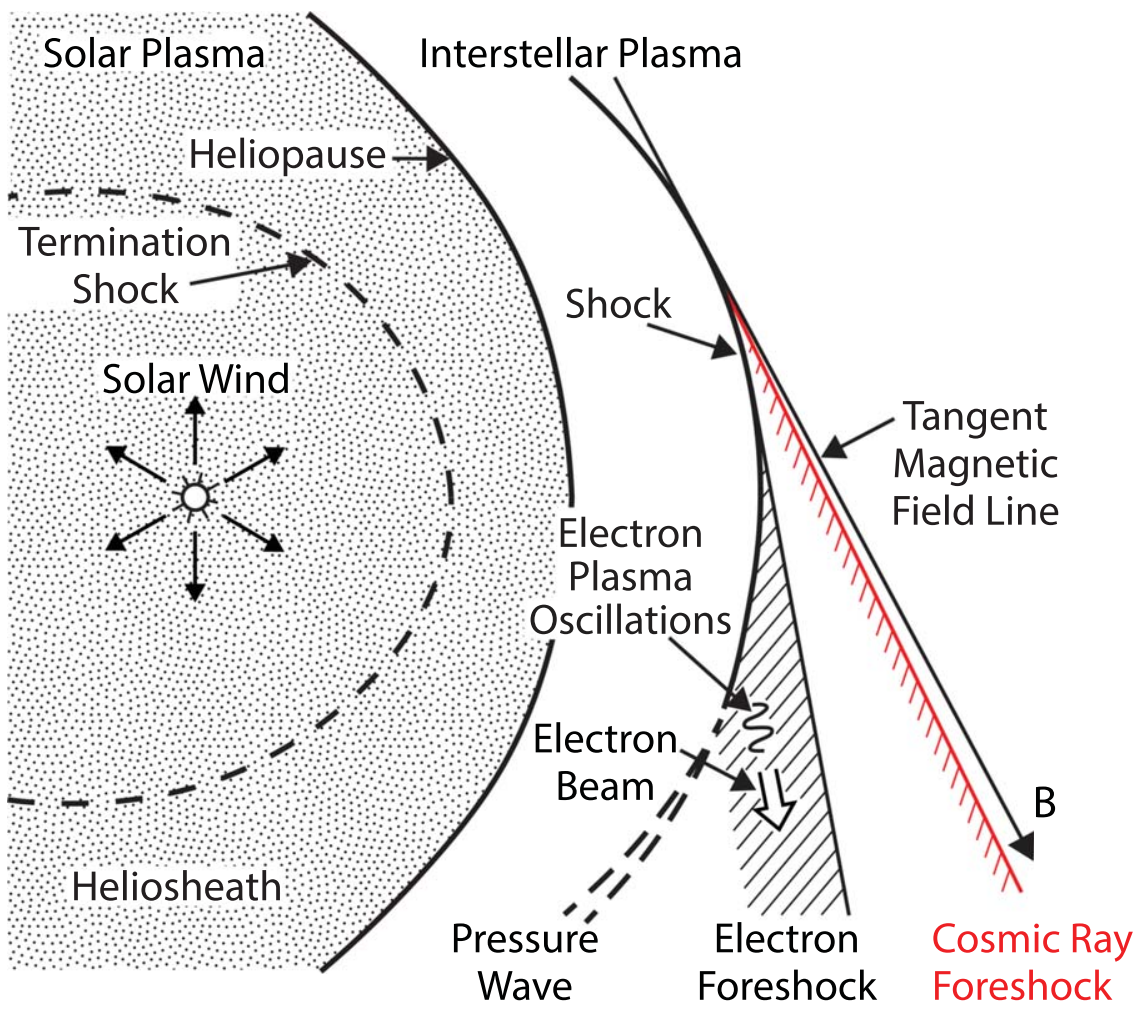

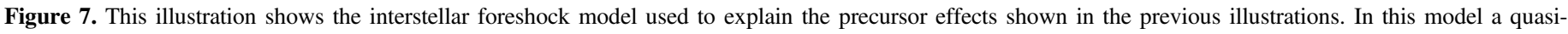

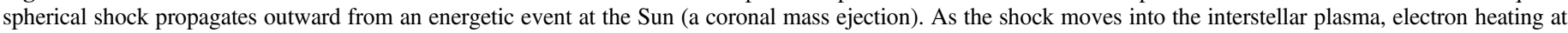

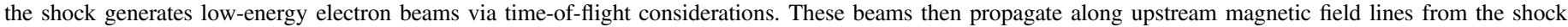

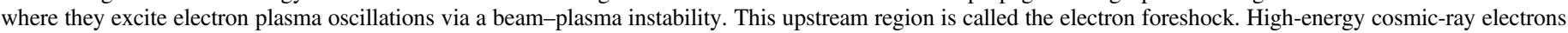

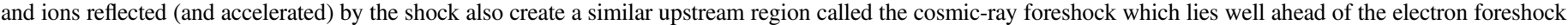
and behind the magnetic field line tangent to the shock.

of the particles involved in each region. In the case of the terrestrial foreshock, the upstream boundary is usually defined by the abrupt onset of electron plasma oscillations. In the interstellar case, the upstream boundary of the electron foreshock is similarly marked by the abrupt onset of electron plasma oscillations, a good example being on day 133 in panel (a) of Figure 4. This boundary is ultimately determined by the positive slope consideration involved in the generation of plasma oscillations. For the cosmic-ray foreshock, the upstream boundary is determined by the speed of light, which is the maximum speed of cosmic-ray electrons and ions reflected by the shock.

We might now ask if there are any significant tests that can be performed to further validate the interstellar foreshock model. It turns out that there two such tests, the first of which comes from the CRS high-energy, HET1 and HET2, telescopes. One of the rates from these high-energy telescopes reports 5-60 MeV ions arriving in two conical fields of view, the boresights of which are perpendicular to each other. The half angles of the fields of view are approximately $20^{\circ}$. The different boresight directions of the two telescopes were originally intended to investigate cosmic-ray anisotropies. Usually the components of the interstellar magnetic field projected along the boresights of the two telescopes have different signs. Using data from these telescopes we can check to see if reflected cosmic-ray ions are coming from the vicinity of the tangent field point, where the magnetic field line through the spacecraft first contacts the shock. During the period from day 111 to 133 of 2014 (see Figure 8), the average angles between the interstellar magnetic field and the boresights of the two telescopes were $137^{\circ}$ for the HET1 field of view and $35^{\circ}$ for the HET2 field of view. Detailed analyses of the geometry of these two fields of view for a quasi-spherical shock arriving from the Sun, as in Figure 7, show that the tangent field point can only be viewed by the HET1 telescope. Figure 8 shows that ions (mostly protons) reflected from the shock are only detected in the HET1 telescope, but not in the HET2 telescope, therefore consistent with a crucial prediction of the interstellar foreshock model.

The second test of the interstellar foreshock model is to estimate the velocity of the low-energy electrons escaping upstream of the shock and see if this velocity is consistent with the energy of electron beams thought to be responsible for driving the electron plasma oscillations (i.e., electrons in the energy range from a few tens of electron volts to several kiloelectron volts). The discovery of bursts of $\sim 5-100 \mathrm{MeV}$ cosmic-ray electrons reflected from the first contact of the shock with the magnetic field line through the spacecraft essentially gives us a $t=0$ reference time for the travel time, $\Delta t$, of electrons from the shock to the spacecraft where they produce local electron plasma oscillations. Since relativistic electrons with energies of $\sim 5-100 \mathrm{MeV}$ are moving very close to the speed of light, it is expected that the travel time for these electrons is essentially negligible (fraction of a day) compared to the observed delay times, $\Delta t=\sim 13-30$ days, for the lowenergy electron beams responsible for the plasma oscillations. The remaining unknown quantity in estimating the speed of these electrons, $v=L_{\mathrm{t}} / \Delta t$, is the distance $L_{\mathrm{t}}$ to the tangent field point. We use two techniques to estimate this distance. The first is based on the geometry shown in Figure 9, where we have 


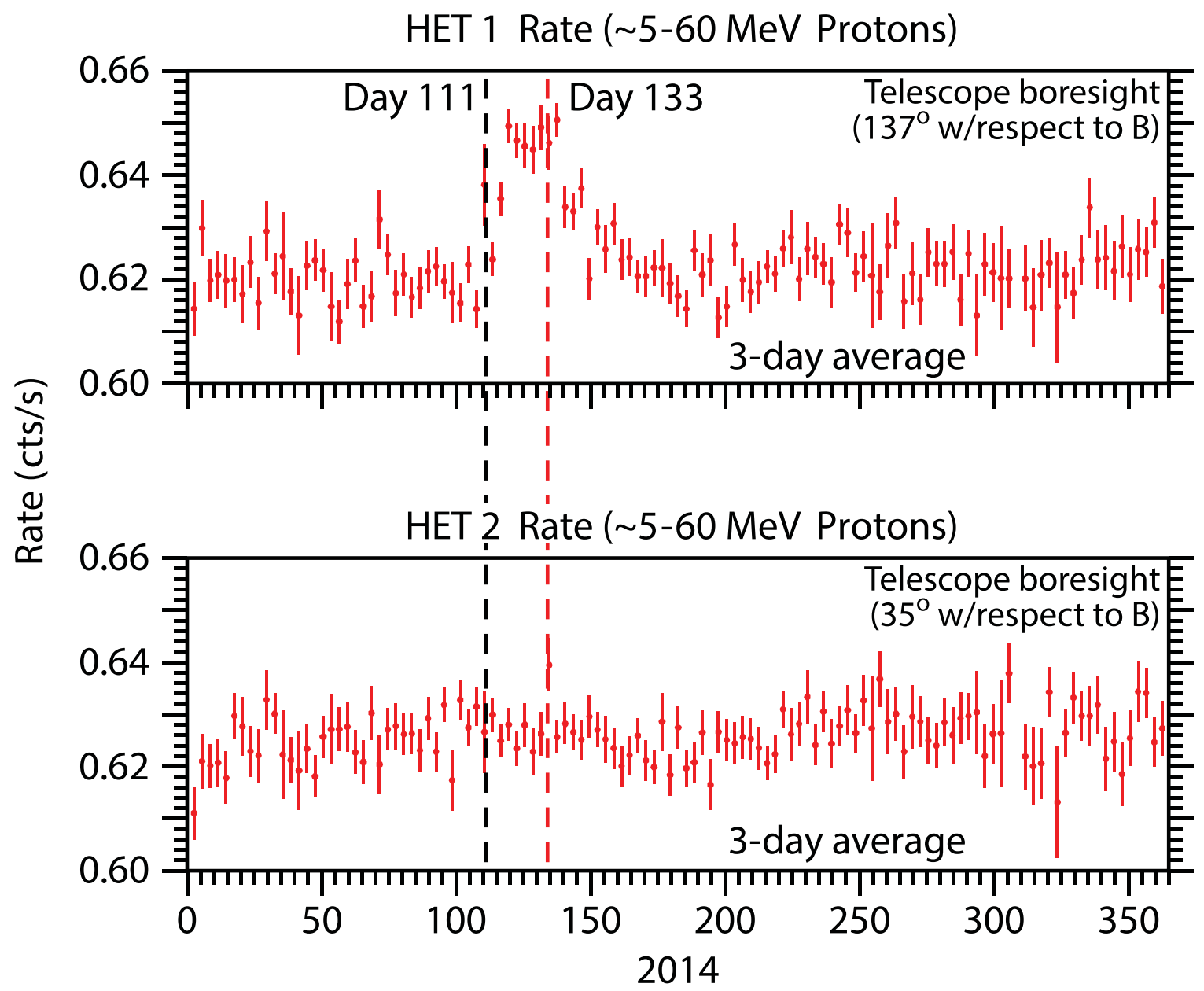

Figure 8. During the day 111 to 113, 2014 event, the average angles between the interstellar magnetic field and the boresights of the two CRS high-energy telescopes were $137^{\circ}$ for the HET1 field of view and $35^{\circ}$ for the HET2 field of view. Protons reflected from the shock are detected by the HET1 telescope, but not by the HET2 telescope. Since the tangent magnetic field point is in the field of view of the HET1 telescope, but not in the HET2 telescope, this observation shows that the reflected particles are coming from the direction of the tangent magnetic field point, a crucial test of the foreshock model.

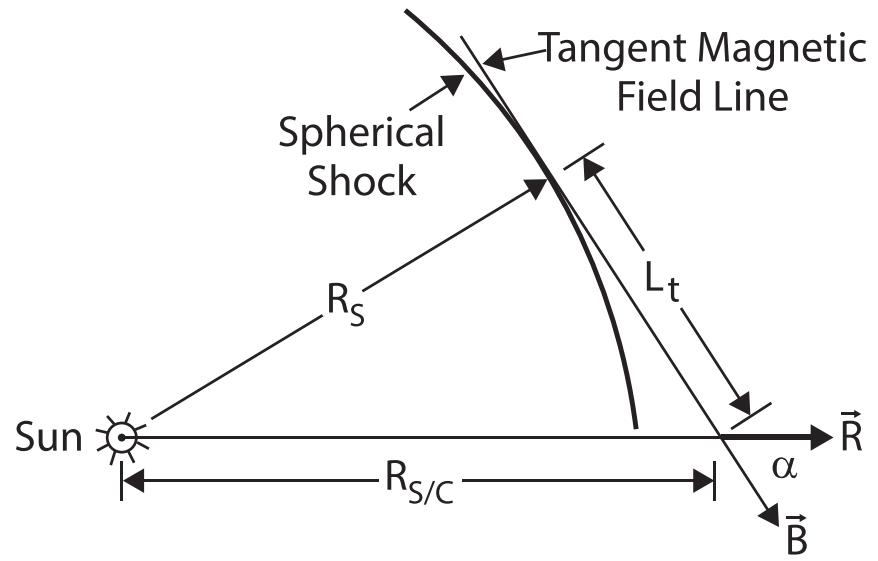

Figure 9. A simple model used to estimate the distance, $L_{\mathrm{t}}$, along the magnetic field line from the spacecraft to the point where the magnetic field first contacts the surface of a shock propagating outward from the Sun. In this model the shock is assumed to be a sphere centered at the Sun, and the magnetic field is assumed to be a straight line. The angle, $\alpha$, between the radial vector from the Sun, $R$, and the magnetic field, $B$, can be determined from the spacecraft magnetometer.

assumed that the shock front is a spherical surface centered at the Sun, and that the interstellar magnetic field is a straight line. Although the spherical approximation for the shock surface is probably not accurate in any given case, the hope is that the results are reasonable when averaged over many cases. By using the magnetic field direction, $\alpha$, measured from the spacecraft magnetometer and the known radial distance of the spacecraft from the Sun, $R_{\mathrm{S} / \mathrm{c}}$, the distance to the tangent point, $L_{\mathrm{t}}$, can be computed for all eight plasma oscillation events shown in Figure 2. This distance is found to vary from $L_{\mathrm{t}}=13.8$ to $50.2 \mathrm{au}$, with an average of $33.7 \mathrm{au}$. The magnetic field components and other parameters used in these calculations are summarized in Table 1.

The second method of estimating $L_{\mathrm{t}}$ is based on measuring the difference in the arrival time of electrons and the ions (assumed to be protons) reflected near the tangent field point. This technique has proven to be difficult in practice, because the time delay is small and the count rates for the particles involved are low, which leads to poor time resolution. However, there is one case, on day 111 of the 2014 February-November event, for which the counting rates are high enough to give reasonably reliable results, see Figure 10. In this case, the time delay between the onset of the reflected electrons and the reflected protons is approximately $\Delta t_{\mathrm{ep}} \sim 8$ days. Since the velocity of the relativistic $\sim 5-100 \mathrm{MeV}$ electrons is close to the speed of light, for the analysis we assume that the difference in the travel time is determined mainly by the reflected protons. To estimate the travel time of 


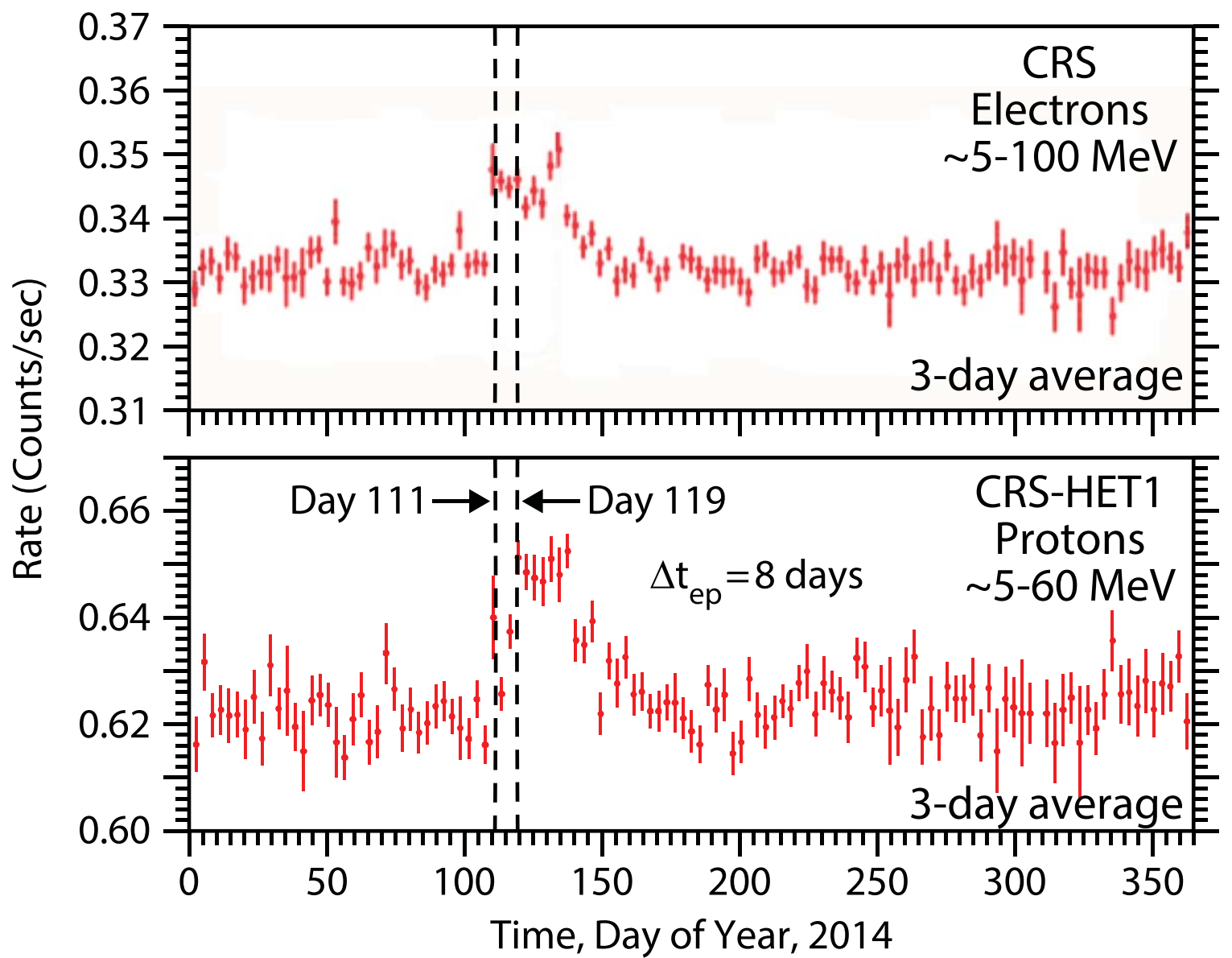

Figure 10. A measurement of the time delay, $\Delta t \approx 8$ days, between the arrival of $\sim 5-100 \mathrm{MeV}$ cosmic-ray electrons and the arrival of $\sim 5-60 \mathrm{MeV}$ ions (assumed to be protons) reflected at the time of first contact of the magnetic field through the spacecraft with a shock of solar origin around day 111, 2014. Using this delay time and the estimated velocity of the particles, the distance to the tangent field point is estimated to be $\sim 39.6$ au. This distance is reasonably consistent with the distances, 13.8-50.2 au (with an average of $33.7 \mathrm{au}$ ), obtained from the simple geometric model in Figure 9 (see Table 1).

Table 1

Distance along the Magnetic Field to the Tangent Point

\begin{tabular}{lcccccc}
\hline \hline Event & Day & $B_{\mathrm{R}}(\mathrm{nT})$ & $B_{\mathrm{T}}(\mathrm{nT})$ & $B_{\mathrm{N}}(\mathrm{nT})$ & $\alpha(\mathrm{deg})$ & $R_{\mathrm{s} / \mathrm{c}}(\mathrm{au})$ \\
\hline 2012 Oct-Nov & 300 & 0.148 & -0.361 & 0.120 & 68.7 & 122.2 \\
2013 Apr-May & 90 & 0.181 & -0.431 & 0.197 & 69.1 & 123.7 \\
2014 Feb-Nov & 230 & 0.185 & -0.407 & 0.159 & 67.0 & 128.7 \\
2015 Sep-Nov & 255 & 0.122 & -0.387 & 0.208 & 74.5 & 44.2 \\
2016 Aug-Oct & 182 & 0.079 & -0.384 & 0.155 & 79.2 & 132.5 \\
2017 Aug-Sep & 226 & 0.097 & -0.385 & 0.198 & 77.4 & 135.4 \\
2018 May-Jun & 153 & 0.040 & -0.374 & 0.174 & 84.4 & 139.4 \\
2019 May-Jun & 142 & 0.071 & -0.368 & 0.159 & 80.0 & 142.2 \\
\hline
\end{tabular}

Note. Magnetic field components are $24 \mathrm{hr}$ averages. This table shows the estimated distances, $L_{\mathrm{t}}$, along the magnetic field from the spacecraft to the point of first contact of the shock for the eight plasma oscillation events illustrated in Figure 2. The calculation of $L_{\mathrm{t}}$ is based on the geometric model in Figure 9. The angle $\alpha$ is computed from the magnetic field components, $B_{\mathrm{R}}, B_{\mathrm{T}}$, and $B_{\mathrm{N}}$ (in nanotesla, nT), as measured by the $\mathrm{V} 1$ magnetometer. The RTN coordinate system has the R unit vector radially outward from the Sun, $T$ parallel to the solar equator (positive in the direction of solar rotation), and $N$ completes the right-hand coordinate system.

the protons we must consider the pitch angle of the protons reflected by the shock. The minimum pitch angle, $\alpha_{0}$, for which reflection can occur is determined by the jump in the magnetic field, $B_{2} / B_{1}$, across the shock. Using the first adiabatic invariant this pitch is given by $\sin ^{2} \alpha_{0}=B_{1} / B_{2}$. For the 2014 event, the $\mathrm{B}$ field ratio across has been determined to be $B_{2} / B_{1} \sim 1.4$ at the spacecraft (Gurnett et al. 2015). Having no other alternative, we assume that this $\mathrm{B}$ field ratio is also comparable to that near the tangent field point. The pitch angle of the reflected proton is then limited to a narrow range of pitch angles, $57.7^{\circ}<\alpha<90^{\circ}$. Because the cosmic-ray proton intensities decrease with increasing energy, to compute the average energy of cosmic-ray protons reflected from the shock, we take the lower limit of the HET1 passband, $\sim 5 \mathrm{MeV}$, to be representative of the energy of the protons being detected. Assuming the average pitch angles of the reflected proton to be about $\left(90^{\circ}+57.7^{\circ}\right) / 2$, and recognizing that the velocity component along the magnetic field line is proportional to cos 
$\alpha$, using the observed time delay, $\Delta t_{\mathrm{ep}}=8$ days, the distance to the tangent field point works out to be $L_{\mathrm{t}}=39.6 \mathrm{au}$. Given the uncertainties involved in this calculation, this distance agrees rather well with the distance to the tangent field point estimated earlier from purely geometric considerations. Using these estimates and an average electron pitch angle of $45^{\circ}$, the velocity of the electron beam works out to be $4100 \mathrm{~km} \mathrm{~s}^{-1}$, which corresponds to an energy of $47.9 \mathrm{eV}$. Note that this velocity is much greater than the $26 \mathrm{~km} \mathrm{~s}^{-1}$ flow velocity of the interstellar medium relative to the Sun (Ajello et al. 1987), so the interstellar plasma flow makes only a minor correction to distance traveled by the electron beam (relative to $L_{\mathrm{t}}$, as estimated above). Considering the wide range of parameters involved in this calculation we estimate that the electron beam energies involved in driving the electron plasma oscillations could be anywhere from about $20-100 \mathrm{eV}$, with average of about $50 \mathrm{eV}$. Although these energies are somewhat low compared to the electron beam energies typically responsible for driving electron plasma oscillations ahead of the terrestrial bow shock, they are comparable to those measured by Bale et al. (1999), 100-150 eV, for the electron beams responsible for Type II solar radio emissions. Therefore, we believe these are reasonable estimates for the energies of the electron beams driving the interstellar electron plasma oscillations. Since the electron temperature in the interstellar medium is low $\left(10^{4} \mathrm{~K}\right)$ when compared to these beam energies, Landau damping is not expected to be a significant factor in limiting the growth of these beam driven electron plasma oscillations.

\section{Conclusion}

In the roughly eight years since V1 reached interstellar space in 2012, ten distinct electron plasma oscillation events have been observed, eight by V1 and two by V2, all of which are believed to be driven by shocks of solar origin propagating into the interstellar plasma. The electron densities inferred from these plasma oscillations have increased from $0.055 \mathrm{~cm}^{-3}$ at $121.6 \mathrm{au}$, shortly after the heliopause crossing, to $0.13 \mathrm{~cm}^{-3}$ for the most recent event at $146 \mathrm{au}$. Whether the density of $0.13 \mathrm{~cm}^{-3}$ represents the asymptotic density of the VLISM, or is a peak and will eventually decrease as the spacecraft proceeds farther out into the interstellar medium, remains to be determined. Some interpretations of the upper cutoff frequency of the band of $\sim 2 \mathrm{kHz}$ radio emissions thought to be trapped within the heliospheric density cavity (Gurnett et al. 1993) suggest that the average density of local interstellar medium could be somewhat less than $0.13 \mathrm{~cm}^{-3}$, perhaps around $0.08 \mathrm{~cm}^{-3}$ (i.e., $f_{\mathrm{p}}=2.5 \mathrm{kHz}$ ). Two recent electron plasma oscillation events detected by V2 shortly after it crossed the heliopause agree closely with the V1 radial density profile, including the presence of a steep plasma density ramp, or boundary layer, in the interstellar plasma immediately beyond the heliopause (Gurnett \& Kurth 2019; Kurth \& Gurnett 2020). Well-defined bursts of high-energy, $\sim 5-100 \mathrm{MeV}$, relativistic electrons are found to consistently precede the plasma oscillation events by from 13 to 30 days. We interpret these bursts of high-energy electrons as arising from the reflection (and acceleration) of relativistic cosmic-ray electrons at the time of first contact of the shock with the interstellar magnetic field line passing through the spacecraft. Using the time delay between this first contact of the approaching shock with the magnetic field line through the spacecraft and the onset time of the electron plasma oscillations, we have for the first time been able to estimate the energies of the upstream electron beams responsible for the electron plasma oscillations. The energies of these electron beams, streaming outward ahead of the interstellar shock, are estimated to be in the range from about 20-100 eV, with an average of about $50 \mathrm{eV}$, low compared to similar electron beams responsible for driving electron plasma oscillations observed ahead of the terrestrial bow shock, but comparable to those responsible for Type II solar radio bursts.

The research at the University of Iowa was supported by NASA through Contract 1622510 with the Jet Propulsion Laboratory. The research at Caltech was supported by NASA award NNN12AA01C, and the research at the Johns Hopkins Applied Physics Laboratory was supported by the Voyager Interstellar Mission under NASA contract NNN06AA01C. The research at Goddard Spaceflight Center was supported by NASA contract 80GSFC19C0012. The Voyager PWS data are regularly archived with the Planetary Data System at https:// pds.nasa.gov.

\section{ORCID iDs}

D. A. Gurnett (iD https://orcid.org/0000-0003-2403-0282

W. S. Kurth (D) https://orcid.org/0000-0002-5471-6202

E. C. Stone (iD https://orcid.org/0000-0002-2010-5462

A. C. Cummings (iD https://orcid.org/0000-0002-3840-7696

S. M. Krimigis (iD https://orcid.org/0000-0003-2781-2386

L. F. Burlaga (1D https://orcid.org/0000-0002-5569-1553

\section{References}

Ajello, J. M., Stewart, A. I., Thomas, G. E., \& Graps, A. 1987, ApJ, 317, 964 Axford, W. I. 1990, in Physics of the Outer Heliosphere, ed. S. Grzedzielski \& D. E. Page (Oxford: Pergamon), 7

Bale, S. D., Reiner, M. J., Bougeret, J.-L., et al. 1999, GeoRL, 26, 1573

Baranov, V. B., \& Malama, Yu. G. 1993, JGR, 98, 15157

Bridge, H. S., Belcher, J. W., Butler, R. J., et al. 1977, SSRv, 21, 259

Burlaga, L. F., Ness, N. F., Berdichevsky, D. B., et al. 2019, NatAs, 3, 1007

Burlaga, L. F., Ness, N. F., Gurnett, D. A., \& Kurth, W. S. 2013a, ApL, 778, L3

Burlaga, L. F., Ness, N. F., \& Stone, E. C. 2013b, Sci, 341, 147

Cairns, I. H., \& Zank, G. P. 2000, in The Outer Heliosphere: The Next Frontier, ed. K. Scherer et al. (Oxford: Pergamon Press), 253

Davis, L. E., Jr. 1955, PhRv, 100, 1440

Filbert, P. C., \& Kellogg, P. J. 1979, JGR, 84, 1369

Frisch, P. C., Redfield, S., \& Slavin, J. D. 2011, ARA\&A, 49, 237

Fuselier, S. A., \& Cairns, I. H. 2013, ApJ, 771, 83

Ginzburg, V. L., \& Zheleznyakov, V. V. 1958, SvA, AJ2, 653

Gurnett, D. A., \& Bhattacharjee, A. 2017, Introduction to Plasma Physics With Space, Laboratory, and Astrophysical Applications (2nd ed.; Cambridge: Cambridge Univ. Press)

Gurnett, D. A., \& Kurth, W. S. 2019, NatAs, 3, 1024

Gurnett, D. A., Kurth, W. S., Allendorf, S. C., \& Poynter, R. L. 1993, Sci, 262, 199

Gurnett, D. A., Kurth, W. S., Burlaga, L. F., \& Ness, N. F. 2013, Sci, 341,1489

Gurnett, D. A., Kurth, W. S., Stone, E. C., et al. 2015, ApJ, 809, 121

Jokipii, J. R., \& Kota, J. 2014, ApL, 794, L4

Krimigis, S. M., Armstrong, T. P., Axford, W. I., et al. 1977, SSRv, 21, 329

Krimigis, S. M., Decker, R. B., Roelof, E. C., et al. 2019, NatAs, 3, 997

Krimigis, S. M., Decker, R. M., Roelof, E. C., et al. 2013, Sci, 341, 144

Kurth, W. S., \& Gurnett, D. A. 2020, ApJL, 900, L1

Kurth, W. S., Gurnett, D. A., Scarf, F. S., \& Poynter, R. L. 1984, Natur, 312, 27

Parker, E. N. 1963, Interplanetary Dynamical Processes (New York: Interscience)

Pogorelov, N. V., Heerikhuisen, J., Roytershteyn, V., et al. 2017, ApJ, 845, 9

Rankin, J. S., Stone, E. C., Cummings, A. C., et al. 2019, ApJ, 873, 46 
Reiner, M. J., Kaiser, M. L., Fainberg, J.-L., \& Stone, R. G. 1998, JGR, 103, 29651

Richardson, J. D., Belcher, J. W., Garcia-Galindo, P., \& Burlaga, L. F. 2019 , NatAs, 3, 1019

Scarf, F. L., \& Gurnett, D. A. 1977, SSRv, 21, 289

Stilwell, D. E., Davis, W. D., Joyce, R. M., et al. 1979, ITNS, 1, 513

Stone, E. C., Cummings, A. C., Heikkila, B. C., \& Lal, N. 2019, NatAs, 3, 1013
Stone, E. C., Cummings, A. C., McDonald, F. B., et al. 2013, Sci, 341, 150

Stone, E. C., Vogt, R. E., McDonald, F. B., et al. 1977, SSRv, 21, 355

Warwick, J. W., Pearce, J. B., Peltzer, R. G., \& Riddle, A. C. 1977, SSRv, 21, 309

Wild, J. P., Murray, J. D., \& Rowe, W. C. 1954, AuJPh, 7, 439

Zank, G. P. 2015, ARA\&A, 53, 449

Zhang, M., \& Pogorelov, N. 2020, ApJ, 895, 1 